\title{
MicroRNA-106a confers cisplatin resistance in non-small cell lung cancer A549 cells by targeting adenosine triphosphatase-binding cassette A1
}

\author{
YANXIN MA ${ }^{1,2}$, XUENAN LI $^{1}$, SONG CHENG $^{1}$, WEI WEI ${ }^{2}$ and YAMING LI ${ }^{1}$ \\ ${ }^{1}$ Department of Nuclear Medicine, The First Affiliated Hospital of China Medical University, Shenyang, Liaoning 110001; \\ ${ }^{2}$ Department of Nuclear Medicine, General Hospital of Daqing Oil Field, Daqing, Heilongjiang 163000, P.R. China
}

Received December 7, 2013; Accepted September 12, 2014

DOI: $10.3892 / \mathrm{mmr} .2014 .2688$

\begin{abstract}
MicroRNAs (miRNAs) have been discovered to have pivotal roles in regulating the drug resistance of various types of human cancer, including cisplatin (DDP) resistance in non-small cell lung cancer (NSCLC). Fewer studies have explored the roles of miR-106a in NSCLC-cell resistance to DDP and its precise molecular mechanism has remained elusive. In the present study, whether miR-106a was able to mediate resistance of the lung cancer cell line A549 to DDP was investigated. Reverse transcription quantitative polymerase chain reaction was used to analyze miR-106a mRNA expression levels. miR-106a expression levels were upregulated in the DDP-resistant cell line A549/DDP compared with its parental cell line, A549. miR-106a-transfection induced DDP-resistance in A549 cells, while repression of miR-106a by anti-miR-106a in A549/DDP resulted in enhanced DDP cytotoxicity. Furthermore, it was discovered that the mechanism of miR-106a-induced DDP resistance involved the expression of adenosine triphosphatase-binding cassette, sub-family A, member 1 (ABCA1), as indicated by transfection of cells with short interfering RNA-ABCA1. The results of the present study suggested a novel mechanism underlying DDP-resistance in NSCLC.
\end{abstract}

\section{Introduction}

Non-small cell lung cancer (NSCLC) is the most common type of lung cancer and is a leading cause of lung cancer-associated mortality worldwide (1-3). Patients with NSCLC are mainly treated using platinum-based chemotherapy, including cisplatin (diaminedichloroplatinum, DDP)

Correspondence to: Professor Yaming Li, Department of Nuclear Medicine, The First Affiliated Hospital of China Medical University, 92 North 2nd Road, Shenyang, Liaoning 110001, P.R. China E-mail: liyaming9968@163.com

Key words: miR-106a, ATP-binding cassette, sub-family A, member 1, drug resistance, diamminedichloroplatinum, A549 treatment (4). However, the continuous infusion or multiple administration of DDP often results in the development of drug resistance, leading to treatment failure (5). Therefore, it is necessary to elucidate the molecular mechanisms underlying DDP resistance in NSCLC. A greater understanding of these mechanisms may aid the identification of novel therapeutic targets for attenuating DDP resistance.

MicroRNAs (miRNAs), small, noncoding regulatory RNAs of 21-25 nucleotides, are critical regulators of post-transcriptional gene expression (6). Alterations in miRNA expression have been demonstrated to be involved in regulating drug resistance in various tumors. A role of miR-451 in regulating resistance of $\mathrm{MCF}-7$ breast cancer cells to chemotherapeutic drug doxorubicin has been reported (7). miR-215 expression has been demonstrated to result in osteosarcoma and colon cancer cell-chemoresistance to methotrexate and Tomudex (8). miRNA-106a (miR-106a) belongs to the miR-17 family and has been reported to confer DDP resistance to esophageal adenocarcinoma cells (9). Huh et al (10) demonstrated that dysregulation of miR-106a expression confers paclitaxel resistance to ovarian cancer cells. A study suggested that anti-miR-106a (specific to miR-106a) induced A549-cell apoptosis and increased the sensitivity of A549 cells to anti-cancer drugs (11). However, the effects of miR-106a and the mechanism through which miR-106a influences DDP-induced apoptosis in NSCLC remain to be elucidated.

The adenosine triphosphatase-binding cassette (ABC) transporter family of genes comprises multi-drug resistance-associated genes, including 48 members classified into seven subfamilies (ABCA-ABCG) in humans (12). The majority of these transporters, including $\mathrm{ABCB} 1$, $\mathrm{ABCC} 2$ and $\mathrm{ABCG} 2$, have been well studied in their capacity for influencing cancer-drug resistance (13-17). The activity of these transporters may therefore influence the efficacy of chemotherapeutic drugs in tumor treatment. Although miR-106a may have an important role in conferring DDP-resistance (18), the mechanisms through which miR-106a regulates $\mathrm{ABC}$ transporter proteins and modulates DDP-induced apoptosis in NSCLC cells remain to be elucidated. The present study therefore aimed to elucidate the mechanism by which miR-106a interacts with ABCA1 and 
modulates DDP resistance in NSCLC A549 cells. The results may indicate a potential therapeutic target for the treatment of NSCLC patients with DDP-resistance.

\section{Materials and methods}

NSCLC A549 and A549/DDP cell culture. A549 cells and A549/DDP cells (Guangzhou Zixiutang Biotechnology Co, Ltd., Guangzhou, China) were cultured in RPMI-1640 medium (Gibco-BRL, Invitrogen Life Technologies, Carlsbad, CA, USA), supplemented with $10 \%$ fetal bovine serum (FBS) and $1 \%$ PS $(100 \mathrm{U} / \mathrm{ml}$ penicillin, $100 \mu \mathrm{g} / \mathrm{ml}$ streptomycin; DingGuo, Beijing, China) and maintained in a humidified incubator with $5 \% \mathrm{CO}_{2}$ at $37^{\circ} \mathrm{C}$. To maintain the DDP-resistant phenotype, DDP was added to the culture media with a final concentration of $1 \mathrm{mg} / \mathrm{ml}$ for A549/DDP cells. A549/DDP cells were cultured for one week in DDP-free medium prior to their experimental use.

Cell viability assay. A549 and A549/DDP cells were plated in 96-well plates at a density of 5,000 cells/well. Eight hours following transfection, cells were treated with various doses of DDP $(0.125,0.25,0.5,1.0,2.0,4.0$ and $8.0 \mathrm{mg} / \mathrm{ml}$; Dinghui Pharmaceutical Factory, Hunan, China) in combination with MTT and were incubated for $4 \mathrm{~h}$ at $37^{\circ} \mathrm{C}$. The cells were subsequently agitated with MTT solvent on an orbital shaker for $10 \mathrm{~min}$ in the dark. The absorbance at $570 \mathrm{~nm}$ was measured using an automatic microplate reader (Ani Labsystems, Ltd. Oy, Vantaa, Finland).

Reverse transcription quantitative polymerase chain reaction (RT-qPCR) analysis of mRNA expression. Total RNA was extracted using RNAiso Plus (Takara Bio, Inc., Otsu, Japan) according to the manufacturer's instructions. cDNA was synthesized using the RevertAid First-Strand cDNA Synthesis kit (Fermentas, Thermo Fisher Scientific, Pittsburgh, PA, USA) according to the manufacturer's instructions. Following the reverse transcription reaction, $\mathrm{qPCR}$ was conducted using the ABI 7300 PCR System (Applied Biosystems Life Technologies, Foster City, CA, USA), using U6 and $\beta$-actin as endogenous controls for data normalization. Primers were as follows: $\beta$-actin forward, 5'-AATCTGGCACCACACCTTCT-3' and reverse, 5'-AGCACAGCCTGGATAGCAAC-3'; ABCA1 forward, 5'-TCTCCAGAGCCAACCTGGCAGCA-3' and reverse, 5'-CCACAGGAGACAGCAGGCTAGCGA-3'.

Transfection ofmiR-106amimic, inhibitorand shortinterfering (si)RNA-ABCA1.ThemiR-106amimic, inhibitoranti-miR-106a and relative control mimic were purchased from GenePharma (Shanghai, China). The sequences were as follows: miR-106a mimic, 5'-GAUGGACGUGACAUUCGUGAAAA-3' and anti-miR-106a, 5'-CUACCUGCACUGUAAGCACUUUU-3'. siRNA-ABCA1 (si-ABCA1) was also purchased from GenePharma. The sequences used in the present study were as follows: siR sense, 5'-GGAUGUACAACGAACAGUACA-3' and antisense, 5'-UACUGUUCGUUGUACAUCCAG-3' (12). In a transfection assay, the cells were transfected with $50 \mathrm{nM}$ miR-106a mimic, anti-miR-106a or si-ABCA1 and siRNA control using Lipofectamine ${ }^{\circledR} 2000$ reagent (Invitrogen Life Technologies, Carlsbad, CA, USA) according to the manufacturer's instructions. The medium was replaced with fresh RPMI-1640 (DingGuo) containing 10\% FBS (DingGuo) and antibiotics $(100 \mathrm{U} / \mathrm{ml}$ penicillin and $100 \mu \mathrm{g} / \mathrm{ml}$ streptomycin) $6 \mathrm{~h}$ post-transfection. For qPCR and western blot analysis, the cells were collected following an additional $48 \mathrm{~h}$ of incubation. Three independent experiments were performed.

Dual luciferase activity assay. The 3'-untranslated region(UTR) of human $A B C A 1$ cDNA containing the putative target site for miR-106a was chemically synthesized and inserted into the pGL3-control vector, downstream of the luciferase gene (Promega, Madison, WI, USA). Cells were plated at $2 \times 10^{5}$ cells/well in 24 -well plates. Subsequently, pGL3-ABCA1-3'-UTR and 80 ng Renilla luciferase-herpes simplex virus-thymidine kinase control reporter vector (Promega) were transfected in combination with miR-106a mimic, anti-miR-106a or controls using Lipofectamine ${ }^{\circledR} 2000$ (Invitrogen Life Technologies) according to the manufacturer's instructions. Luciferase activity was measured using the Dual Luciferase Reporter Assay system (Promega). Firefly luciferase activity was normalized to Renilla luciferase activity for each transfected well.

Western blot analysis. To obtain whole cell lysates, the cell pellet was resuspended in whole cell lysis buffer $(10 \mathrm{mM}$ Tris at $\mathrm{pH} 7.4,1 \mathrm{mM} \mathrm{NaF}, 1 \mathrm{mM} \mathrm{Na} \mathrm{VO}_{4}, 1 \mathrm{mM}$ phenylmethanesulfonyl fluoride, $0.1 \%$ sodium dodecyl sulfate, $1 \%$ Triton X-100 and the protease inhibitor cocktail; Nanjing chemistry company, Nanjing, China) and was subsequently lysed by freezing-thawing followed by sonication. The first antibody was rabbit polyclonal anti-ABCA1 (EMD Millipore, Billerica, MA, USA; 1:100 dilution) and anti- $\beta$-actin antibody (Abcam, San Francisco, CA, USA; 1:1,000 dilution). The secondary antibody was goat anti-rabbit immunoglobulin $\mathrm{G}$, conjugated with horseradish peroxidase at a dilution of 1:1,000. The bound antibodies were detected using Enhanced Chemiluminescence Plus Western Blotting Detection system (GE Healthcare, Little Chalfont, UK). $\beta$-actin was used as an internal control to normalize ABCA1 expression levels. Representative images from one of three independent experiments are exhibited. The blotting band intensity was quantified using Image-J software (http://imagej.nih.gov/ij/).

Annexin V and propidium iodide (PI) staining. Enumeration of apoptotic cells was performed using fluorescein isothiocyanate (FITC)-conjugated Annexin V and PI (BD Pharmingen, San Diego, CA, USA). Cells were washed twice in cold $1 \mathrm{X}$ PBS and resuspended in Annexin V-binding buffer (BD Pharmingen) at a concentration of $3 \times 10^{6}$ cells $/ \mathrm{ml}$. This suspension $(100 \mu \mathrm{l})$ was stained with $5 \mu \mathrm{l}$ Annexin V-FITC and $5 \mu \mathrm{l}$ PI. The cells were gently vortexed and incubated for $15 \mathrm{~min}$ at room temperature in the dark. Following the addition of $400 \mu 1$ binding buffer to each tube, cells were analyzed by flow cytometry (Cytomics FC500; Beckman Coulter, Miami, FL, USA).

Statistical analysis. Values are presented as the mean + standard deviation and experiments were repeated three times. The difference was determined by two-tailed Student's t-tests 
A

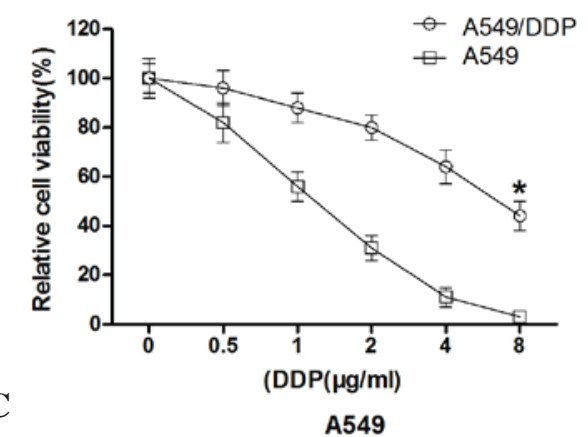

C

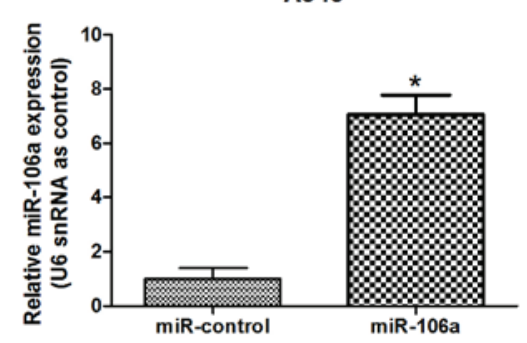

$\mathbf{E}$

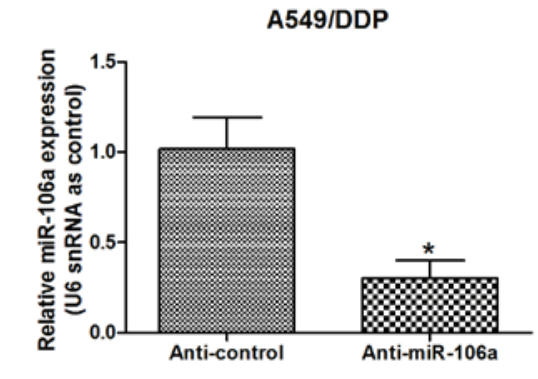

B
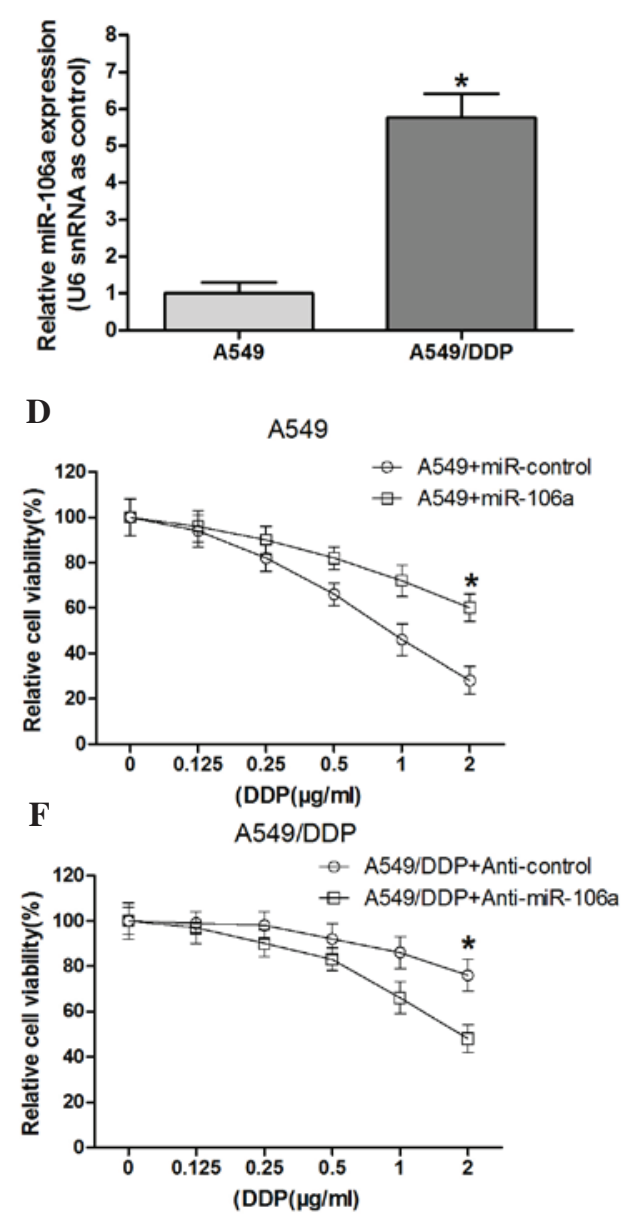

Figure 1. miR-106a is upregulated in DDP-resistant A549/DDP cells and involved in conferring DDP resistance to A549 cells. (A) Survival curves of A549/DDP and A549 cells. Cells were treated with various doses of DDP $(0.125,0.25,0.5,1.0,2.0,4.0$ and $8.0 \mathrm{mg} / \mathrm{ml})$. Following $48 \mathrm{~h}$ of incubation, cell viability was measured by MTT assay. (B) RT-qPCR analysis of miR-106a mRNA expression levels in A549 and A549/DDP cells. U6 snRNA was used as a loading control. (C and E) qRT-PCR indicated that miR-106a expression levels were significantly increased or decreased in the cells transfected with the miR-106a mimic or anti-miR-106a in A549 cells or A549/DDP cells, respectively. (D and F) Transfected A549 cells or A549/DDP cells were incubated with various doses of DDP $(0.125,0.25,0.5,1.0$ and $2.0 \mathrm{mg} / \mathrm{ml})$ for $48 \mathrm{~h}$ prior to MTT assay to determine cell viability. ${ }^{*} \mathrm{P}<0.05$. DDP, cisplatin; RT-qPCR, reverse transcription quantitative polymerase chain reaction; snRNA, small nuclear RNA; miR, micro RNA; mRNA, messenger RNA.

using GraphPad 5.0 software (GraphPad Software, Inc., La Jolla, CA, USA). $\mathrm{P}<0.05$ was considered to indicate a statistically significant difference between values.

\section{Results}

miR-106a is upregulated in DDP-resistant A549/DDP cells and involved in conferring DDP-resistance to A549 cells. Survival curves of the A549 and A549/DDP cells in response to various doses of DDP were detected (Fig. 1A). The results revealed that the A549/DDP cell lines exhibited greater resistance to DDP compared with that of the A549 cell line. Subsequently, the involvement of miR-106a in DDP-resistant A549/DDP cells was investigated using RT-qPCR. The results indicated that miR-106a expression levels were higher in A549/DDP cells than in A549 cells (Fig. 1B), which suggested that miR-106a may be associated with DDP resistance in NSCLC cells, which was in agreement with a previous study (10). Furthermore, in order to investigate the association between miR-106a and DDP resistance in A549 cells, the effect of overexpression of miR-106a on A549 cells was evaluated. RT-qPCR revealed that transfection with the miR-106a mimic significantly increased miR-106a mRNA expression levels, which suggested that miR-106a was efficiently transfected into the cells (Fig. 1C and D). The miR-106a mimic-transfected A549 cells had a significantly higher survival rate than that of the negative control (miR-control) group. The effect of miR-106a repression on A549/DDP cells was also tested. The relative results (Fig. 1E and F) indicated that the A549/DDP cells transfected with anti-miR-106a had a significantly lower survival rate than that of the control group. These results demonstrated that miR-106a confers DDP-resistance in A549 cells and knockdown of miR-106a was able to moderately sensitize A549/DDP cells to DDP.

$A B C A 1$ is a candidate target gene of miR-106a. As miR-106a has a pivotal function in conferring DDP resistance of NSCLC A549 cells, it is important to explore the target genes of miR-106a involved in the DDP-resistance mechanism. To 


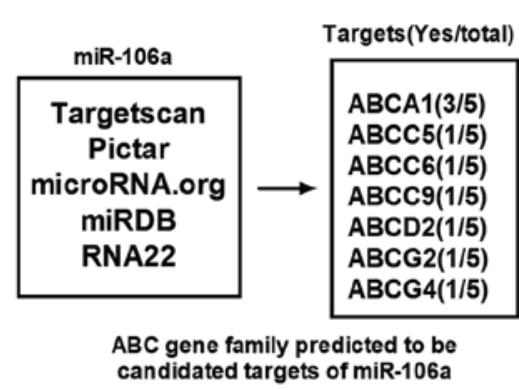

B

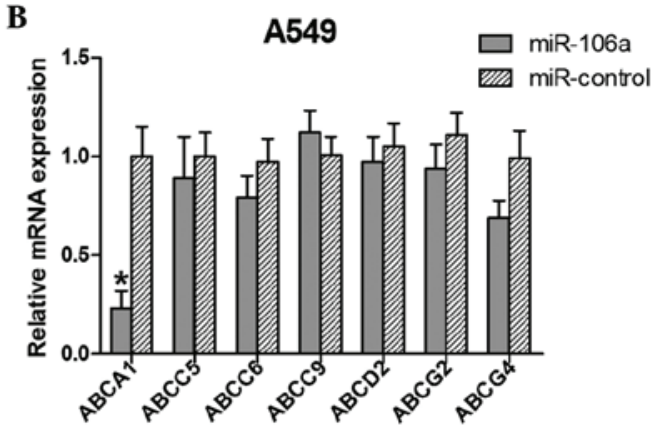

C

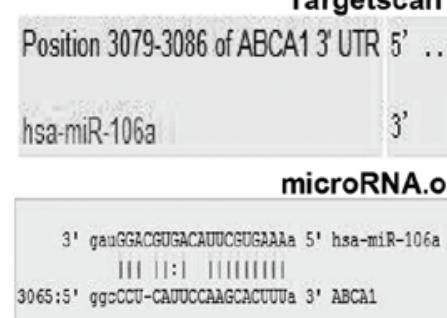

Targetscan

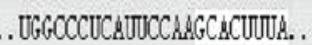

|| ||||

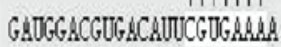

mirSVR score: $\quad-0.6212$
D

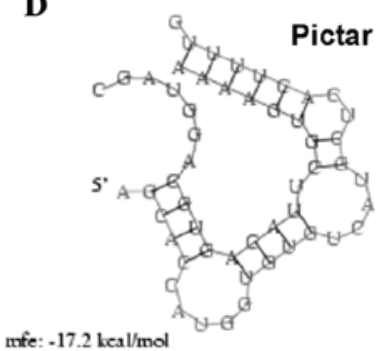

Figure 2. ABCA1 is a potential miR-106a target. (A) Seven members of the ABC gene transporter family were identified as potential miR-106a targets using bioinformatics. (B) mRNA levels of ABC gene transporters were measured by quantitative reverse-transcription polymerase chain reaction in miR-106a mimictransfected A549 cells. U6 small nuclear RNA was set as loading control. * $\mathrm{P}<0.05$ compared with control. (C and D) Algorithms between miR-106a and the 3'UTR of ABCA1 in micoRNA.org, Targetscan and Pictar software. ABC, adenosine triphosphatase-binding cassette; miR, microRNA; UTR, untranslated region; hsa, Homo sapiens; mRNA, messenger RNA.

determine which $\mathrm{ABC}$ transporter genes may be regulated by miR-106a, six miRNA target prediction algorithms were utilized in order to identify the target genes of miR-106a. A list of seven $\mathrm{ABC}$ transporter genes was identified, including $A B C A 1, A B C C 5, A B C C 6, A B C C 9, A B C D 2, A B C G 2$ and $A B C G 4$ (Fig. 2A). Among these genes, $A B C A 1$ had the greatest frequency (three of the five algorithms predicted that the 3'UTR of $A B C A 1$ contained the putative binding site of miR-106a). A549 cells were subsequently transfected with miR-106a and RT-qPCR was performed in order to detect the expression levels of the seven candidate target genes of miR-106a. Of the potential target genes identified, $A B C A 1$ was selected, as it was demonstrated to have the lowest expression levels in the transfected A549 cells (Fig. 2B). The alignments of miR-106a with the ABCA1 3'UTR insert predicted by Targetscan, Pictar and microRNA.org software are illustrated in Fig. 2C and D.

$A B C A 1$ is a direct target of miR-106a. To determine whether miR-106a directly regulates $A B C A 1$ by binding to the 3 'UTR of the target gene, a luciferase reporter assay was performed. Wild-type and mutant ABCA1 3'UTR sequences which contained the miR-106a binding site were constructed and inserted into pGL3 vectors (Fig. 3A). The reporter construct and miR-106a mimics were transfected into the DDP-sensitive cell line (A549). Concurrently, the reporter construct and anti-miR-106a were transfected into the DDP-resistant A549/DDP cell line. When miR-106a was overexpressed in A549 cells, luciferase expression levels were significantly lower than those in the miR-control group. However, the luciferase intensity in the mutant ABCA1 3'UTR cells was unaffected by miR-106a transfection (Fig. 3B). In analogy, repression of miR-106a caused an increase in luciferase levels in DPP-resistant
A

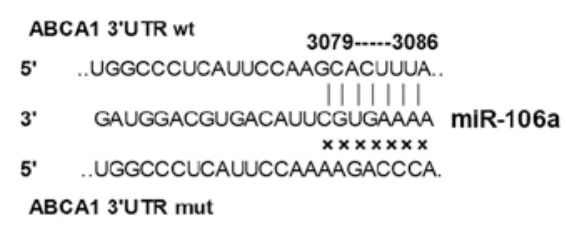

B

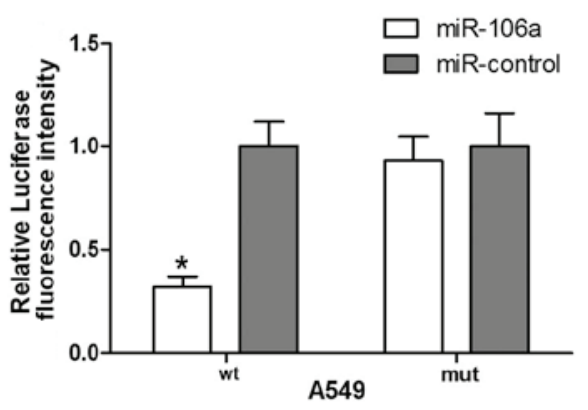

C

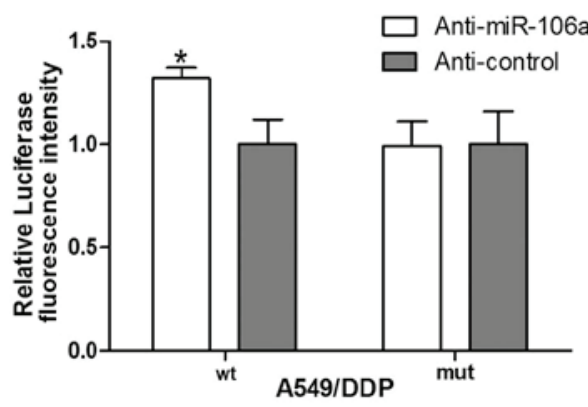

Figure 3. miR-106a targets ABCA1 3'UTR in A549 and A549/DDP cells (A) Algorithms between miR-106a and the 3'UTR of ABCA1 and the mutant ABCA1 3'UTR. (B and C) A luciferase reporter assay was performed to detect the effect of miR-106a on luciferase intensity, controlled by the 3'UTR of ABCA1. ${ }^{*} \mathrm{P}<0.05$ compared with control. ABCA1, adenosine triphosphatase-binding cassette sub-family A, member 1; UTR, untranslated region; miR, microRNA; DPP, cisplatin; mut, mutant; wt, wild-type. 
A

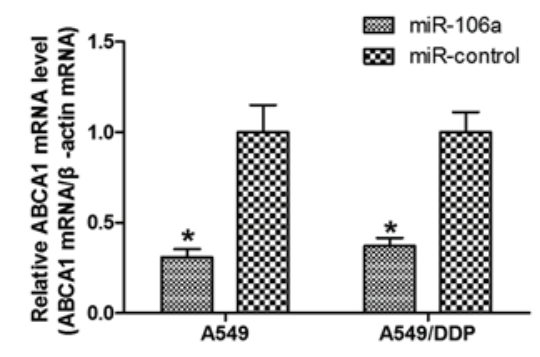

B

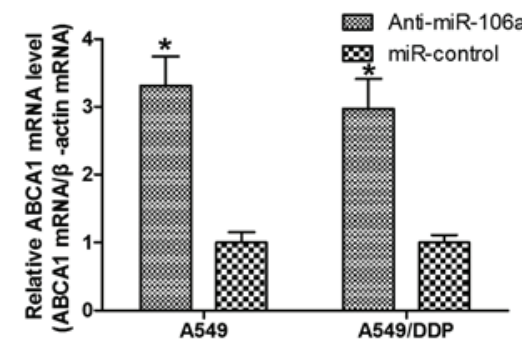

C

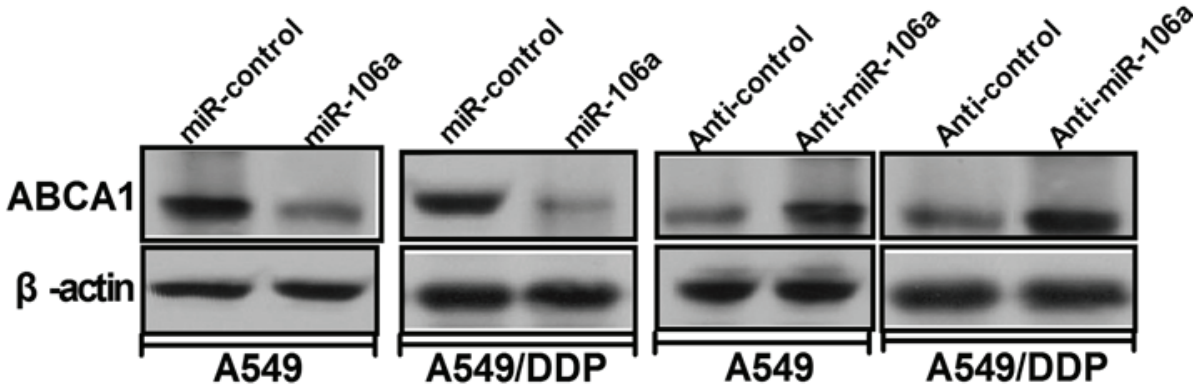

D

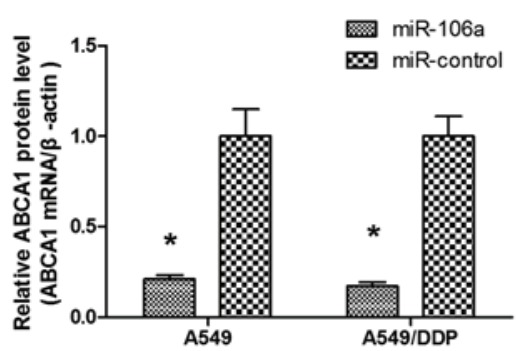

$\mathbf{E}$

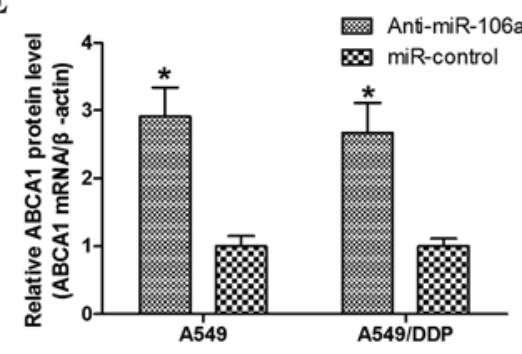

Figure 4. miR-106a regulates ABCA1 mRNA and protein expression in A549 and A549/DDP cells. (A and B) quantitative reverse transcription polymerase chain reaction was performed to evaluate the effects of miR-106a and anti-miR-106a on ABCA1 mRNA expression. $\beta$-actin was used as a loading control. (C-E) Western bloting was performed to detect the effect of miR-106a and anti-miR-106a on protein levels of ABCA1. $\beta$-actin was used as a loading control. "P<0.05 compared with control. ABCA1, adenosine triphosphatase-binding cassette sub-family A, member 1; mRNA, messenger RNA; miR, micro RNA; DDP, cisplatin.

cells, while mutant cells were unaffected (Fig. 3C). It was therefore concluded that $A B C A 1$ was a direct target of miR-106a.

miR-106a downregulates ABCA1 mRNA and protein expression levels. RT-qPCR and western blotting assays were performed in order to determine the regulation of endogenous $A B C A 1$ expression levels by miR-106a. The results demonstrated that overexpression of miR-106a significantly decreased ABCA1 mRNA and protein expression levels in A549 and A549/DDP cells (P<0.05; Fig. 4A, C and D). Simultaneously, repression of miR-106a significantly increased ABCA1 mRNA and protein expression levels in A549 and A549/DDP cells $(\mathrm{P}<0.05$; Fig. $4 \mathrm{~B}, \mathrm{C}$ and $\mathrm{E})$. These results suggested that miR-106a negatively regulated $A B C A 1$ expression in A549 and A549/DDP cells.

ABCA1 is an important signaling molecule in miR-106a-regulated DDP-resistance in A549/DDP cells. The results of the present study demonstrated that miR-106a overexpression induced DDP-resistance, miR-106a knockdown rescued DDP sensitivity and miR-106a was able to directly target ABCA1, a membrane transporter involved in drug uptake. To determine whether $A B C A 1$ had a key role in miR-106a-regulated DDP resistance, ABCA1 siRNA or si-negative control (NC) was transfected into anti-miR-106a transfected A549/DDP cells and the cell survival rate under various concentrations of DDP was evaluated. Western blot analysis indicated that ABCA1 siRNA effectively reduced the protein expression levels of ABCA1 (Fig. 5A). ABCA1 knockdown significantly increased the survival rate of the A549/DDP cells transfected with anti-miR-106a compared with that of the si-NC group (Fig. 5B), which suggested that miR-106a modulated DDP resistance in A549/DDP cells by targeting ABCA1. To confirm this mechanism, an additional rescue assay was performed using flow cytometry. As indicated in Fig. 5C and D, ABCA1 knockdown significantly decreased the apoptotic rate of A549/DDP cells transfected with anti-miR-106a compared with that of the si-NC group.

\section{Discussion}

Chemoresistance is one of the most significant obstacles in the successful treatment of lung cancer (5). Extensive studies have demonstrated that miRNAs may act as regulators of chemosensitivity in addition to regulating oncogenes or tumor-suppressor genes in various types of human cancer $(19,20)$. With respect to lung cancer, miR-31 was reported to inhibit cisplatin-induced cell apoptosis by regulating the drug transporter ABCB9 (21). 
A

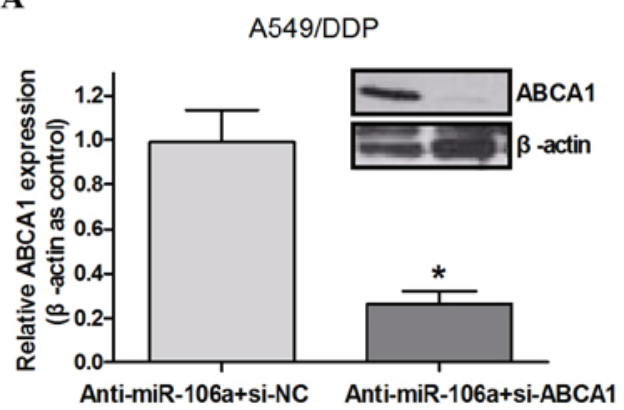

$\mathbf{C}$

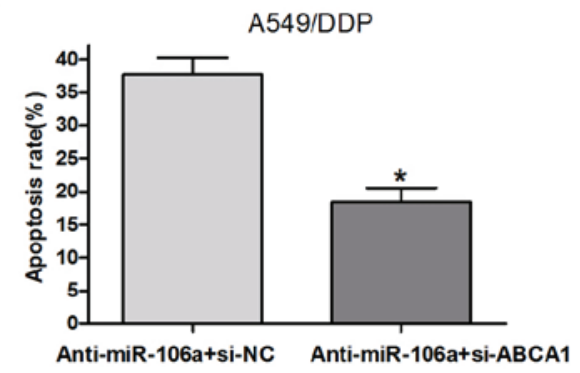

B

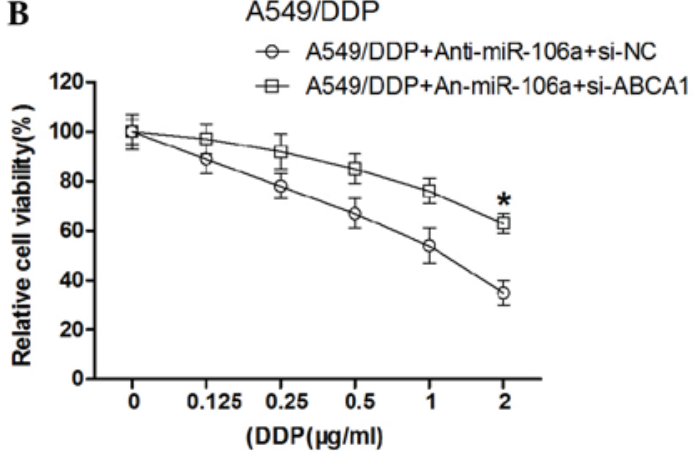

D

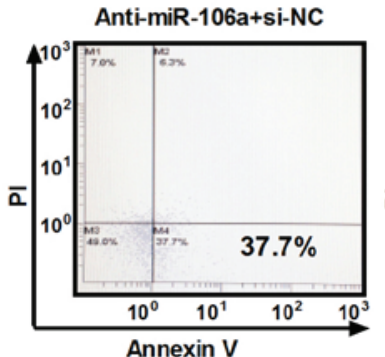

Figure 5. ABCA1 has an important role in miR-106a-regulated DDP-resistance in A549/DDP cells. (A) ABCA1 protein levels in miR-106a inhibitor-transfected A549/DDP cells following treatment with ABCA1 siRNA or siRNA-NC. (B) A549/DDP cells were treated with various doses of DDP $(0.125,0.25,0.5,1.0$ and $2.0 \mathrm{mg} / \mathrm{ml}$ ) $48 \mathrm{~h}$ post-transfection. Cell viability was determined by MTT assay. (C) miR-106a-inhibited A549/DDP cells were treated with ABCA1 siRNA or a siRNA-NC, $48 \mathrm{~h}$ following transfection. Apoptotic rate was determined by flow cytometry. (D) Representative figures. "P<0.05 compared with NC. mRNA, messenger RNA; siRNA, short interfering RNA; miR, micro RNA; DDP, cisplatin; PI, propidium iodide; ABCA1, adenosine triphosphatase-binding cassette sub-family A, member 1 ; NC, negative control.

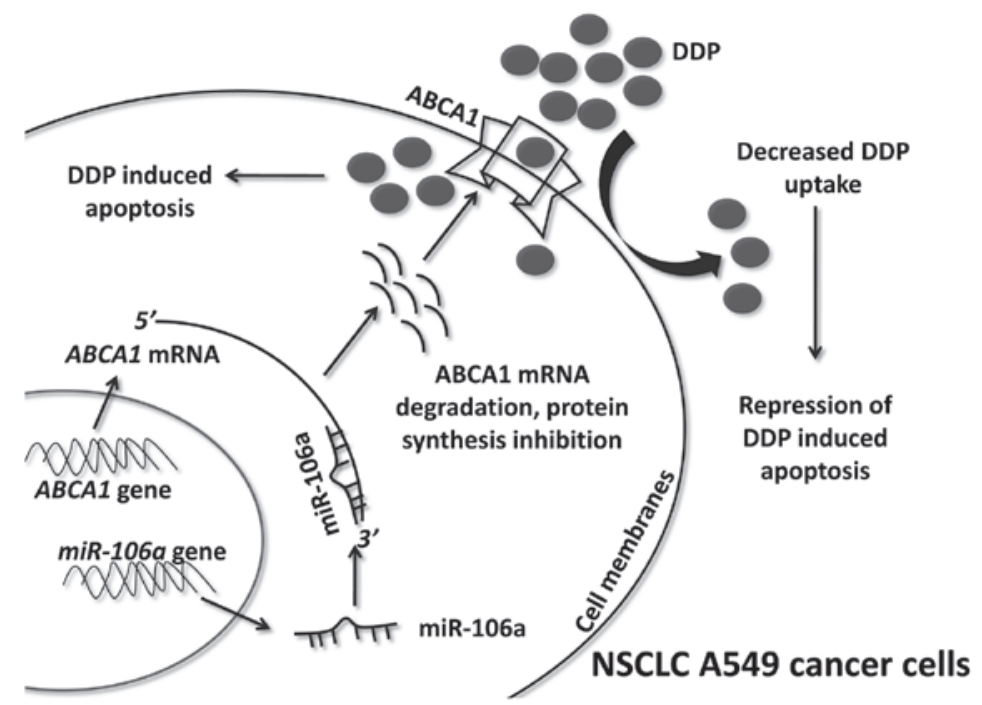

Figure 6. Model of miR-106a-mediated cisplatin resistance in NSCLC A549 cells. miR-106a inhibits ABCA1 expression and subsequently reduces DDP uptake, resulting in repression of DDP-induced apoptosis. ABCA1, adenosine triphosphatase-binding cassette sub-family A, member 1; mRNA, messenger RNA; miR, micro RNA; DDP, cisplatin, NSCLC, non-small cell lung cancer.

A recent study indicated that the upregulation of miR-92b influenced the cisplatin-chemosensitivity phenotype of lung cancer cells by targeting PTEN (22). Another study demonstrated that miR-495 enhanced the sensitivity of NSCLC cells to platinum by modulation of copper-transporting P-type adenosine triphosphatase A (23). However, few studies have investigated the role of miR-106a and its target genes in lung cancer-cell drug resistance, although its association with cancer has been well characterized. In the present study, the expression levels of miR-106a in DDP-resistant A549 cells (A549/DDP) were detected by RT-qPCR and the results indicated that the upregulation of miR-106a expression levels were associated with DDP resistance. It was also confirmed that the modulation of miR-106a in A549/DDP cells resensitized them to DDP.

The $\mathrm{ABC}$ gene families have been well characterized in their association with drug or multi-drug resistance in various types 
of human cancers, including P-glycoprotein $(A B C B 1)(24,25)$. In the present study, three computational algorithms were used to predict putative targets of miR-106a. These algorithms identified seven members of the ABC gene family which had putative target sites in their 3'UTR. ABCAl was validated as the most significant target of miR-106a in lung cancer cell lines by reporter-gene assay and western blot analysis. In several cell lines, functional targets of miR-106a have been verified, including SLC2A3, p130 and FASTK (26-28). The present study, for the first time, suggests that miR-106a directly targeted the $A B C A 1$ 3'UTR and regulated its mRNA and protein expression levels.

$A B C A 1$ is a member of the $\mathrm{ABC}$ transporter family and has an important role in cellular and body metabolism. It has been reported that cancer-specific $A B C A 1$ hypermethylation and loss of protein expression result in high intracellular cholesterol levels and therefore contribute to the creation of an environment conducive to tumor progression (29). Another study demonstrated an anti-cancer function of the cholesterol exporter ABCA1 in human cancer cells and reported that reconstitution of $A B C A 1$ expression inhibited tumor formation (30). $A B C A 1$ has also been demonstrated to have important roles in resistance to curcumin in M14 melanoma cells (31). These reports support the results of the present study in suggesting that $A B C A 1$, as an important $\mathrm{ABC}$ gene transporter, mediated miR-106a-induced DDP resistance in A549 cells. The present study additionally established a rescue assay to confirm this hypothesis. miR-106a-repressed A549/DDP cells were transfected with si-ABCA1, which demonstrated that the viability of A549/DDP cells was recovered in the cells in the si-ABCA1 group compared with those in the control group. Furthermore, the high apoptotic rate of A549/DDP cells caused by miR-106a knockdown was attenuated by si-ABCA1. These results associated miR-106a, which has an anti-apoptotic role, and ABCA1, which acts as a tumor suppressor, with DDP resistance in A549 cells. It was therefore hypothesized that miR-106a was upregulated in a DDP-resistant NSCLC cell line compared with a corresponding DDP-sensitive NSCLC cell line (A549/DDP compared to A549). miR-106a upregulation suppressed ABCA1 mRNA and protein expression levels. Thus, the inhibition of ABCA1 resulted in decreased DDP uptake in A549 cells. These factors contributed to the miR-106a-induced DDP resistance of A549 cells (Fig. 6).

Further study will reveal that miR-106a is an anti-apoptotic factor in other NSCLC cell lines. The present study provided the first evidence, to the best of our knowledge, that miR-106a has an important role in conferring DDP-resistance by targeting $A B C A 1$ in A549 cell lines and thus exhibits anti-apoptotic properties. The present study highlighted the potentially important role of miR-106a in the development of DDP resistance and suggested that miR-106a may serve as a biomarker which may be used to predict patient response to DDP in NSCLC.

\section{References}

1. Siegel R, DeSantis C, Virgo K, Stein K, Mariotto A, Smith T, Cooper D, Gansler T, Lerro C, Fedewa S, et al: Cancer treatment and survivorship statistics, 2012. CA Cancer J Clin 62: 220-241, 2012.

2. Jemal A, Center MM, DeSantis C and Ward EM: Global patterns of cancer incidence and mortality rates and trends. Cancer Epidemiol Biomarkers Prev 19: 1893-1907, 2010.
3. Saika K and Machii R: Cancer mortality attributable to tobacco by region based on the WHO Global Report. Jpn J Clin Oncol 42: 771-772, 2012.

4. Stinchcombe TE and West HL: Maintenance therapy in non-small-cell lung cancer. Lancet 374: 1398-1400, 2009.

5. Chang A: Chemotherapy, chemoresistance and the changing treatment landscape for NSCLC. Lung cancer. 71: 3-10, 2011.

6. Bartel DP: MicroRNAs: genomics, biogenesis, mechanism, and function. Cell 116: 281-297, 2004.

7. Kovalchuk O, Filkowski J, Meservy J, Ilnytskyy Y, Tryndyak VP, Chekhun VF and Pogribny IP: Involvement of microRNA-451 in resistance of the MCF-7 breast cancer cells to chemotherapeutic drug doxorubicin. Mol Cancer Ther 7: 2152-2159, 2008.

8. Song B, Wang Y, Titmus MA, Botchkina G, Formentini A, Kornmann $\mathrm{M}$ and Ju J: Molecular mechanism of chemoresistance by miR-215 in osteosarcoma and colon cancer cells. Mol Cancer 9 : 96, 2010.

9. Hummel R, Hussey DJ and Haier J: MicroRNAs: predictors and modifiers of chemo- and radiotherapy in different tumour types. Eur J Cancer 46: 298-311, 2010.

10. Huh JH, Kim TH, Kim K, Song JA, Jung YJ, Jeong JY, Lee MJ, Kim YK, Lee DH and An HJ: Dysregulation of miR-106a and miR-591 confers paclitaxel resistance to ovarian cancer. $\mathrm{Br}$ J Cancer 109: 452-461, 2013.

11. Wang PY, Li YJ, Zhang S, Li ZL, Yue Z, Xie N and Xie SY: Regulating A549 cells growth by ASO inhibiting miRNA expression. Mol Cell Biochem 339: 163-171, 2010.

12. Locher KP: Structure and mechanism of ABC transporters. Curr Opin Struct Biol 14: 426-431, 2004

13. Iwasaki H, Okabe T, Takara K, Yoshida Y, Hanashiro K and Oku H: Down-regulation of lipids transporter ABCA1 increases the cytotoxicity of nitidine. Cancer Chemother Pharmacol 66: 953-959, 2010.

14. Borst P, Evers R, Kool M and Wijnholds J: A family of drug transporters: the multidrug resistance-associated proteins. J Natl Cancer Inst 92: 1295-1302, 2000.

15. Gottesman MM, Fojo T and Bates SE: Multidrug resistance in cancer: role of ATP-dependent transporters. Nat Rev Cancer 2: 48-58, 2002

16. Cole SP, Bhardwaj G, Gerlach JH, Mackie JE, Grant CE, Almquist KC, Stewart AJ, Kurz EU, Duncan AM and Deeley RG: Overexpression of a transporter gene in a multidrug-resistant human lung cancer cell line. Science 258: 1650-1654, 1992.

17. Deeley RG and Cole SP: Function, evolution and structure of multidrug resistance protein (MRP). Semin Cancer Biol 8: 193-204, 1997.

18. Fang Y, Shen H, Li H et, al. miR-106a confers cisplatin resistance by regulating PTEN/Akt pathway in gastric cancer cells. Acta Biochim Biophys Sin (Shanghai). 2013. 45(11):963-72.

19. Gong C, Yao Y, Wang Y, Liu B, Wu W, Chen J, Su F, Yao H and Song E: Up-regulation of miR-21 mediates resistance to trastuzumab therapy for breast cancer. J Biol Chem 286: 19127-19137, 2011.

20. Suh SS, Yoo JY, Nuovo GJ, Jeon YJ, Kim S, Lee TJ, Kim T, Bakàcs A, Alder H, Kaur B, et al: MicroR NAs/TP53 feedback circuitry in glioblastoma multiforme. Proc Natl Acad Sci USA 109: 5316-5321, 2012.

21. Dong Z, Zhong Z, Yang L, Wang S and Gong Z: MicroRNA-31 inhibits cisplatin-induced apoptosis in non-small cell lung cancer cells by regulating the drug transporter ABCB9. Cancer Lett 343: 249-257, 2014.

22. Li Y, Li L, Guan Y, Liu X, Meng Q and Guo Q: MiR-92b regulates the cell growth, cisplatin chemosensitivity of A549 non small cell lung cancer cell line and target PTEN. Biochem Biophys Res Commun 440: 604-610, 2013.

23. Song L, Li Y, Li W, Wu S and Li Z: miR-495 enhances the sensitivity of non-small cell lung cancer cells to platinum by modulation of copper-transporting P-type adenosine triphosphatase A (ATP7A). J Cell Biochem 115: 1234-1242, 2014.

24. Gillet JP, Efferth T and Remacle J: Chemotherapy-induced resistance by ATP-binding cassette transporter genes. Biochim Biophys Acta 1775: 237-262, 2007.

25. Yamagishi T, Sahni S, Sharp DM, Arvind A, Jansson PJ and Richardson DR: P-glycoprotein mediates drug resistance via a novel mechanism involving lysosomal sequestration. J Biol Chem 288: 31761-31771, 2013.

26. Dai DW, Lu Q, Wang LX, Zhao WY, Cao YQ, Li YN, Han GS, Liu JM and Yue ZJ: Decreased miR-106a inhibits glioma cell glucose uptake and proliferation by targeting SLC2A3 in GBM. BMC Cancer 13: 478, 2013. 
27. Liu Z, Gersbach E, Zhang X, Xu X, Dong R, Lee P, Liu J, Kong B, Shao C and Wei JJ: miR-106a represses the Rb tumor suppressor p130 to regulate cellular proliferation and differentiation in high-grade serous ovarian carcinoma. Mol Cancer Res 11: 1314-1325, 2013.

28. Zhi F, Zhou G, Shao N, Xia X, Shi Y, Wang Q, Zhang Y, Wang R, Xue L, Wang S, et al: miR-106a-5p inhibits the proliferation and migration of astrocytoma cells and promotes apoptosis by targeting FASTK. PLoS One 8: e72390, 2013.
29. Lee BH, Taylor MG, Robinet P, et al: Dysregulation of cholesterol homeostasis in human prostate cancer through loss of ABCA1. Cancer Res 73: 1211-1218, 2013.

30. Smith B and Land H: Anticancer activity of the cholesterol exporter ABCA1 gene. Cell Rep 2: 580-590, 2012.

31. Bachmeier BE, Iancu CM, Killian PH, Kronski E, Mirisola V, Angelini G, Jochum M, Nerlich AG and Pfeffer U: Overexpression of the ATP binding cassette gene ABCA1 determines resistance to Curcumin in M14 melanoma cells. Mol Cancer 8: 129, 2009. 